\title{
A De Bruijn-Erdős theorem for chordal graphs
}

\author{
Laurent Beaudou \\ Adrian Bondy \\ Université Blaise Pascal \\ Clermont-Ferrand, France \\ Université Paris 6 \\ Paris, France \\ laurent . beaudou@univ-bpclermont.fr \\ adrian.bondy@sfr.fr \\ Xiaomin Chen \\ Shanghai Jianshi Ltd \\ Shanghai, P.R. China \\ gougle@gmail.com
Maria Chudnovsky*
Columbia University
New York, U.S.A. \\ mchudnov@columbia.edu \\ Nicolas Fraiman \\ McGill University \\ Montreal, Canada \\ nfraiman@gmail.com

\section{Ehsan Chiniforooshan} \\ Google Kitchener-Waterloo \\ Waterloo, Canada \\ chiniforooshan@alumni.uwaterloo.ca \\ Vašek Chvátal ${ }^{\dagger}$ \\ Concordia University \\ Montreal, Canada \\ chvatal@cse. concordia.ca \\ Yori Zwols \\ Concordia University \\ Montreal, Canada \\ yzwols@gmail.com
}

Submitted: Jul 1, 2013; Accepted: Mar 11, 2015; Published: Mar 23, 2015

Mathematics Subject Classifications: 05C12, 52C10, 05D05

\begin{abstract}
A special case of a combinatorial theorem of De Bruijn and Erdős asserts that every noncollinear set of $n$ points in the plane determines at least $n$ distinct lines. Chen and Chvátal suggested a possible generalization of this assertion in metric spaces with appropriately defined lines. We prove this generalization in all metric spaces induced by connected chordal graphs.
\end{abstract}

Keywords: Combinatorial geometry; Metric space; Extremal combinatorics

*Partially supported by NSF grants DMS-1001091 and IIS-1117631.

${ }^{\dagger}$ Canada Research Chair in Discrete Mathematics. 


\section{Introduction}

It is well known that

(i) every noncollinear set of $n$ points in the plane determines at least $n$ distinct lines.

As noted by Erdős [12], theorem (i) is a corollary of the Sylvester-Gallai theorem (asserting that, for every noncollinear set $S$ of finitely many points in the plane, some line goes through precisely two points of $S$ ); it is also a special case of a combinatorial theorem proved later by De Bruijn and Erdős [11].

Theorem (i) involves neither measurement of distances nor measurement of angles: the only notion employed here is incidence of points and lines. Such theorems are a part of ordered geometry [7], which is built around the ternary relation of betweenness: point $b$ is said to lie between points $a$ and $c$ if $b$ is an interior point of the line segment with endpoints $a$ and $c$. It is customary to write $[a b c]$ for the statement that $b$ lies between $a$ and $c$. In this notation, a line $\overline{u v}$ is defined - for any two distinct points $u$ and $v$ - as

$$
\{u, v\} \cup\{p:[p u v] \vee[u p v] \vee[u v p]\} .
$$

In terms of the Euclidean metric dist, we have

$$
[a b c] \Leftrightarrow a, b, c \text { are three distinct points and } \operatorname{dist}(a, b)+\operatorname{dist}(b, c)=\operatorname{dist}(a, c) \text {. }
$$

In an arbitrary metric space, equivalence (2) defines the ternary relation of metric betweenness introduced in [14] and further studied in [1, 3, 8]; in turn, (1) defines the line $\overline{u v}$ for any two distinct points $u$ and $v$ in the metric space. The resulting family of lines may have strange properties. For instance, a line can be a proper subset of another: in the metric space with points $u, v, x, y, z$ and

$$
\begin{aligned}
& \operatorname{dist}(u, v)=\operatorname{dist}(v, x)=\operatorname{dist}(x, y)=\operatorname{dist}(y, z)=\operatorname{dist}(z, u)=1, \\
& \operatorname{dist}(u, x)=\operatorname{dist}(v, y)=\operatorname{dist}(x, z)=\operatorname{dist}(y, u)=\operatorname{dist}(z, v)=2,
\end{aligned}
$$

we have

$$
\overline{v y}=\{v, x, y\} \quad \text { and } \quad \overline{x y}=\{v, x, y, z\} .
$$

Chen [4] proved, using a definition of $\overline{u v}$ different from (1), that the Sylvester-Gallai theorem generalizes in the framework of metric spaces. Chen and Chvátal [5] suggested that theorem (i), too, might generalize in this framework:

(ii) True or false? Every metric space on $n$ points, where $n \geqslant 2$, either has at least $n$ distinct lines or else has a line that consists of all $n$ points.

They proved that

- every metric space on $n$ points either has at least $\lg n$ distinct lines or else has a line that consists of all $n$ points 
and noted that the lower bound $\lg n$ can be improved to $\lg n+\frac{1}{2} \lg \lg n+\frac{1}{2} \lg \frac{\pi}{2}-o(1)$. (Here, as usual, $\lg x$ stands for $\log _{2} x$.)

Every connected undirected graph induces a metric space on its vertex set, where $\operatorname{dist}(u, v)$ is the familiar graph-theoretic distance between vertices $u$ and $v$, defined as the smallest number of edges in a path from $u$ to $v$. (Some people call this the 'hop distance'.) Chiniforooshan and Chvátal [6] proved that

- every metric space induced by a connected graph on $n$ vertices either has $\Omega\left(n^{2 / 7}\right)$ distinct lines or else has a line that consists of all $n$ vertices;

we will prove that the answer to (ii) is 'true' for all metric spaces induced by connected chordal graphs. (We follow the graph-theoretic terminology of Bondy and Murty [2]. In particular, a chordal graph is a graph that contains no induced cycle of length four or more.)

Theorem 1. Every metric space induced by a connected chordal graph on $n$ vertices, where $n \geqslant 2$, either has at least $n$ distinct lines or else has a line that consists of all $n$ vertices.

\section{The proof}

Given an undirected graph, let us write $[a b c]$ to mean that $a, b, c$ are three distinct vertices such that $\operatorname{dist}(a, b)+\operatorname{dist}(b, c)=\operatorname{dist}(a, c)$; this is equivalent to saying that $b$ is an interior vertex of a shortest path from $a$ to $c$.

Lemma 2. Let $s, x, y$ be vertices in a finite chordal graph such that [sxy]. If $\overline{s x}=\overline{s y}$, then $x$ is a cut vertex separating $s$ and $y$.

Proof. The set of all vertices $u$ such that $\operatorname{dist}(s, u)=\operatorname{dist}(s, x)$ separates $s$ and $y$. Among all its subsets that separate $s$ and $y$, choose a minimal one and call it $C$. Since $x$ is an interior vertex of a shortest path from $s$ to $y$, it belongs to $C$. To prove that $C$ includes no other vertex, assume, to the contrary, that $C$ includes a vertex $u$ other than $x$.

Our graph with $C$ removed has distinct connected components $S$ and $Y$ such that $s \in S$ and $y \in Y$; the minimality of $C$ guarantees that each of its vertices has at least one neighbour in $S$ and at least one neighbour in $Y$. Since each of $u$ and $x$ has at least one neighbour in $S$, there is a path from $u$ to $x$ with at least one interior vertex and with all interior vertices in $S$. Let $P$ be a shortest such path; note that $P$ has no chords except possibly the chord $u x$. Similarly, there is a path $Q$ from $u$ to $x$ with at least one interior vertex, and with all interior vertices in $Y$, that has no chords except possibly the chord $u x$. The union of $P$ and $Q$ is a cycle of length at least four; since this cycle must have a chord, vertices $u$ and $x$ must be adjacent. In turn, the union of $Q$ and $u x$ is a chordless cycle, and so $Q$ has precisely two edges. This means that some vertex $v$ in $Y$ is adjacent to both $u$ and $x$. (Similarly, some vertex in $S$ is adjacent to both $u$ and $x$; however, this 
fact is irrelevant to our argument.)

Write $i=\operatorname{dist}(s, x)$ and $j=\operatorname{dist}(x, y)$. Since all vertices $t$ with $\operatorname{dist}(s, t)<i$ belong to $S$ and since $v$ has no neighbours in $S$, we must have $\operatorname{dist}(s, v)>i$; since $\operatorname{dist}(x, v)=1$, we conclude that $\operatorname{dist}(s, v)=i+1$ and that $v \in \overline{s x}$. Since $\overline{s x}=\overline{s y}$, it follows that $v \in \overline{s y}$. Since $\operatorname{dist}(v, x)=1$ and $\operatorname{dist}(x, y)=j$, we have $\operatorname{dist}(v, y) \leqslant j+1$. From $\operatorname{dist}(s, v)=i+1, \operatorname{dist}(s, y)=i+j, \operatorname{dist}(v, y) \leqslant j+1, i \geqslant 1, j \geqslant 1$, and $v \in \overline{s y}$, we deduce that $\operatorname{dist}(v, y)=j-1$.

Since $\operatorname{dist}(u, v)=1$, it follows that $\operatorname{dist}(u, y) \leqslant j ; \operatorname{since} \operatorname{dist}(s, u)=i$ and $\operatorname{dist}(s, y)=$ $i+j$, we conclude that $\operatorname{dist}(u, y)=j$ and $u \in \overline{s y}$. Since $\operatorname{dist}(s, u)=i, \operatorname{dist}(s, x)=i$, and $\operatorname{dist}(u, x)=1$, we have $u \notin \overline{s x}$. But then $\overline{s x} \neq \overline{s y}$, a contradiction.

A vertex of a graph is called simplicial if its neighbours are pairwise adjacent.

Lemma 3. Let s, $x, y$ be three distinct vertices in a finite connected chordal graph. If $s$ is simplicial and $\overline{s x}=\overline{s y}$, then $\overline{x y}$ consists of all the vertices of the graph.

Proof. Since $\overline{s x}=\overline{s y}$, we have $y \in \overline{s x}$, and so $[y s x]$ or $[s y x]$ or [sxy]; since $s$ is simplicial, $[y s x]$ is excluded; switching $x$ and $y$ if necessary, we may assume that [sxy]. Given an arbitrary vertex $u$, we have to prove that $u \in \overline{x y}$. Let $P$ be a shortest path from $s$ to $u$ and let $Q$ be a shortest path from $u$ to $y$. Lemma 2 guarantees that $x$ is a cut vertex separating $s$ and $y$, and so the concatenation of $P$ and $Q$ must pass through $x$. This means that $[s x u]$ or $[u x y]$ (or both). If $[u x y]$, then $u \in \overline{x y}$; to complete the proof, we may assume that $[s x u]$, and so $u \in \overline{s x}$.

Since $\overline{s x}=\overline{s y}$, we have [usy] or [suy] or [syu]; since $s$ is simplicial, [usy] is excluded. If $[s u y]$, then $[s x u]$ implies $[x u y]$; if $[s y u]$, then $[s x y]$ implies $[x y u]$; in either case, $u \in \overline{x y}$.

Proof of Theorem 1. Consider a connected chordal graph on $n$ vertices where $n \geqslant 2$. By a theorem of Dirac [10, Theorem 4], this graph has at least two simplicial vertices; choose one of them and call it $s$. We may assume that the lines $\overline{s z}$ with $z \neq s$ are pairwise distinct (else some line consists of all $n$ vertices by Lemma 3 ). Since the graph is connected and has at least two vertices, $s$ has at least one neighbour; choose one and call it $u$. If $u$ is the only neighbour of $s$, then every path from $s$ to another vertex must pass through $u$, and so $\overline{s u}$ consists of all $n$ vertices. If $s$ has a neighbour $v$ other than $u$, then line $\overline{u v}$ is distinct from all of the $n-1$ lines $\overline{s z}$ with $z \neq s$ : since $s, u, v$ are pairwise adjacent, we have $s \notin \overline{u v}$.

\section{Related theorems}

In Theorem 1, 'connected chordal graph' can be replaced by 'connected bipartite graph':

- every metric space induced by a connected bipartite graph on $n$ vertices, where $n \geqslant 2$, has a line that consists of all $n$ vertices. 
In fact, $\overline{x y}$ consists of all $n$ vertices whenever $x$ and $y$ are adjacent. To prove this, consider an arbitrary vertex $u$. Since the graph is bipartite, $\operatorname{dist}(u, x)$ and $\operatorname{dist}(u, y)$ have distinct parities; since $\operatorname{dist}(x, y)=1$, they differ by at most one. We conclude that $\operatorname{dist}(u, x)$ and $\operatorname{dist}(u, y)$ differ by precisely one, and so $u \in \overline{x y}$.

In Theorem 1, 'connected chordal graph' can be also replaced by 'graph of diameter two': Chvátal [9] proved that

- every metric space on $n$ points where $n \geqslant 2$ and each nonzero distance equals 1 or 2 either has at least $n$ distinct lines or else has a line that consists of all $n$ vertices.

Kantor and Patkós [13] proved that

- if no two of $n$ points in the plane share their $x$ - or $y$-coordinate, then these $n$ points with the $L_{1}$ metric either induce at least $n$ distinct lines or else they induce a line that consists of all of them.

(For sets of $n$ points in the plane that are allowed to share their coordinates, [13] provides a weaker conclusion: these $n$ points with the $L_{1}$ metric either induce at least $n / 37$ distinct lines or else they induce a line that consists of all of them.)

\section{Acknowledgment}

The work whose results are reported here began at a workshop held at Concordia University in June 2011. We are grateful to the Canada Research Chairs program for its generous support of this workshop. We also thank Luc Devroye, François Genest, and Mark Goldsmith for their participation in the workshop and for stimulating conversations.

\section{References}

[1] L.M. Blumenthal, Theory and Applications of Distance Geometry, Oxford University Press, Oxford, 1953.

[2] J.A. Bondy, and U.S.R. Murty, Graph Theory, Springer, New York, 2008.

[3] H. Busemann, The Geometry of Geodesics, Academic Press, New York, 1955.

[4] X. Chen, The Sylvester-Chvátal theorem, Discrete $\&$ Computational Geometry 35 (2006), 193-199.

[5] X. Chen and V. Chvátal, Problems related to a de Bruijn-Erdős theorem, Discrete Applied Mathematics 156 (2008), 2101-2108.

[6] E. Chiniforooshan and V. Chvátal, A de Bruijn-Erdős theorem and metric spaces, Discrete Mathematics \&6 Theoretical Computer Science 13 (2011), 67-74.

[7] H.S.M. Coxeter, Introduction to Geometry, Wiley, New York, 1961.

[8] V. Chvátal, Sylvester-Gallai theorem and metric betweenness, Discrete 86 Computational Geometry 31 (2004), 175-195. 
[9] V. Chvátal, A de Bruijn-Erdős theorem for 1-2 metric spaces, Czechoslovak Mathematical Journal 64 (2014), 45-51.

[10] G.A. Dirac, On rigid circuit graphs, Abh. Math. Sem. Univ. Hamburg 25 (1961), 71-76.

[11] N.G. De Bruijn and P. Erdős, On a combinatorial problem, Indagationes Mathematicae 10 (1948), 421-423.

[12] P. Erdős, Three point collinearity, American Mathematical Monthly 50 (1943), Problem 4065, p. 65. Solutions in Vol. 51 (1944), 169-171.

[13] I. Kantor and B. Patkós, Towards a de Bruijn-Erdős theorem in the $L_{1}$-metric, Discrete $\&$ Computational Geometry 49 (2013), 659-670.

[14] K. Menger, Untersuchungen über allgemeine Metrik, Mathematische Annalen 100 (1928), 75-163. 